\title{
Shock Compression Modeling of Distended Mixtures
}

\author{
Gregg Fenton $^{1} \cdot$ Dennis Grady $^{1} \cdot$ Tracy $_{\text {Vogler }}^{2}$
}

Received: 23 January 2015/Accepted: 15 April 2015/Published online: 23 April 2015

(C) Society for Experimental Mechanics, Inc 2015

\begin{abstract}
Development of material models to describe the thermodynamic shock states of distended mixtures is motivated by the need to understand how these materials respond over large compression ranges starting from low-pressure mechanical crush, extending into extreme thermodynamic states, and subsequent release to low-pressure. A material modeling approach is presented, which is comprised of a thermodynamically consistent equation-of-state (EOS) within a mixture-modeling framework. This modeling approach enables the investigator to describe the dynamic response of distended mixtures over a large compression range. The EOS model is applied to shock Hugoniot data on tungsten carbide, tantalum pentoxide, and calcite-water mixtures.
\end{abstract}

Keywords Equation of state - Porous materials - Theory/ Modeling/Simulation · Shock compaction

\section{Introduction}

The material modeling approach described herein represents a combination of a framework that describes the

Gregg Fenton

gfenton@ara.com

Dennis Grady

dgrady@ara.com

Tracy Vogler

tjvogle@sandia.gov

1 Applied Research Associates, 4300 San Mateo Blvd. NE, Albuquerque, NM 87110, USA

2 Sandia National Laboratory, 7011 East Ave., MS 9042, Livermore, CA 94550, USA low-pressure compaction of a heterogeneous mixture of component materials and an equilibrium high-pressure equation of state (EOS). This model is called the enthalpy-based-shock-compression (EBSC) model. The EBSC model simulates the compaction behavior of a mixture from mechanical crush through extreme pressure loadings.

The low-pressure crush response of the EBSC model is implemented within the $\mathrm{p}-\lambda$ model framework [1]. Originally, the $\mathrm{p}-\lambda$ model was built only for low-pressure mechanical crush of porous mixtures and is suitable for distended mixtures of powdered solids (base matrix material) and interstitial fluids (pore volume material) such as water, air, or vacuum $[1,2]$. The $\mathrm{p}-\lambda$ model is an engineering approach designed to address mixtures of components and multiple states of compaction (e.g., uncompacted to fully compacted). The mixtures of interest will inherently have components with widely disparate densities, compliancy, and strengths.

The EBSC model concept is illustrated in Fig. 1. This concept shows how the $\mathrm{p}-\lambda$ model transitions into the high-pressure equilibrium response of the mixture. The high-pressure response is addressed by the use of a phenomenological enthalpy based equation of state. The enthalpy based approach for distended material was proposed by Rice and Walsh [3] and this method circumvents the analytic complications of backward leaning and multi-valued Hugoniot states common to shock compression of highly distended solids. Extreme shock heating can lead to volume expansions that render the Hugoniot backward leaning (volume increasing with increasing pressure). There are also cases for which the Hugoniot is multi-valued (exhibiting several pressure states corresponding to the same specific volume). 


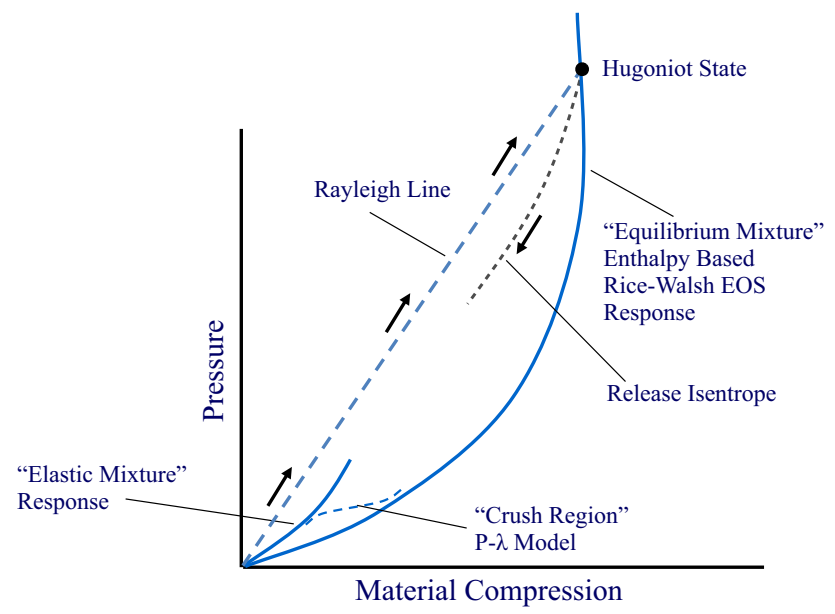

Fig. 1 The EBSC model is illustrated in the pressure-compression plane, showing the boundaries within which the model responds

\section{Low-Pressure Crush}

The $\mathrm{p}-\lambda$ material compaction model is based on an additive superposition of specific volumes as shown in Eq. (1). The $v_{e}(p)$ and $v_{h}(p)$ terms are pressure dependent specific volume relationships for the elastic initially distended media (elastic iso-strain) and the fully compacted mixture (iso-pressure), respectively. The $\lambda$ represents the compaction fraction of the mixture between the elastic isostrain state and the iso-pressure state.

$v(p)=(1-\lambda(p)) v_{e}(p)+\lambda(p) v_{h}(p)$

The iso-strain regime (elastic compression) occurs during the initial compaction of the material. The iso-strain compression relation is nearly linear and proportional to the porous material bulk modulus. The iso-pressure regime (pressure equilibrated) occurs after full compaction is achieved. A full description of the $\mathrm{p}-\lambda$ model, low pressure crush, compaction transition, and definition of Eq. (1) is found in Grady et al. [1].

A graphical representation of the superposition relationship is illustrated in Fig. 2. The $\lambda$ parameter is a pressure dependent function ranging from zero for the initial distended media to unity for the fully compacted. The functional form convenient for $\lambda$ is based on a Weibull statistical strength of materials concept,

$\lambda(p)=1-e^{-(p / \sigma)^{n}}$,

where $\sigma$ and $n$ are the material compaction parameters. The pressure scale is provided by $\sigma$ and is determined by the crush strength of the mixture. The $n$ parameter represents the homogeneity of the compaction and determines the abruptness of the transition to iso-pressure response. $n$ acts like a compaction-hardening exponent. The $\lambda$ also has a

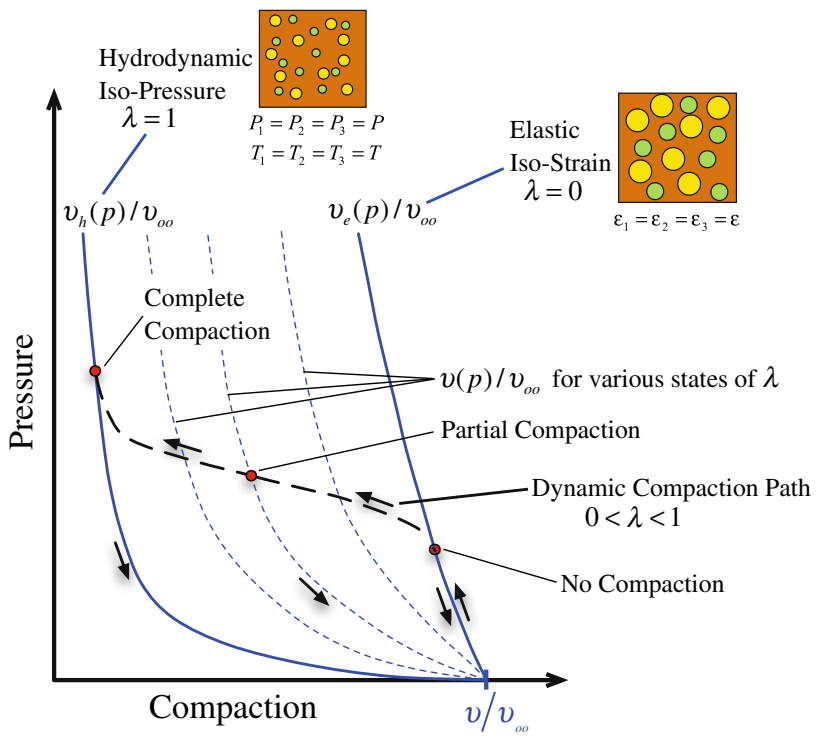

Fig. 2 The p- $\lambda$ model concept is illustrated in the pressurecompaction plane, showing the boundaries of the model. The actual compaction state may exist between the bounding iso-strain and isopressure curves and is determined by computing the $\lambda$ parameter

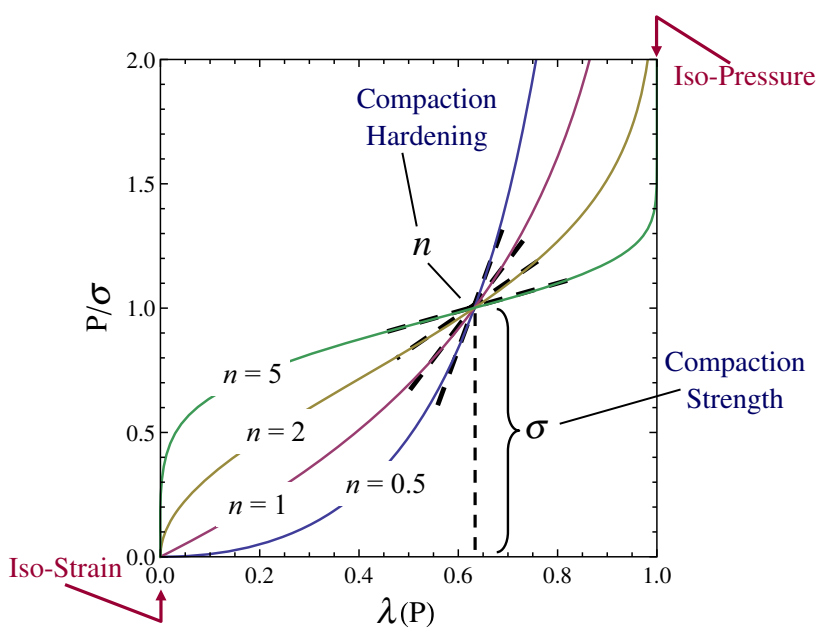

Fig. 3 The $\lambda$ function is plotted to illustrate similarities to a material stress-strain relationship

characteristic where an increase in $\lambda$ is irreversible, so once a material is compacted, that state of compaction is maintained.

A functional illustration of the $\lambda$ parameter is shown in Fig. 3. Material hardness or resistance to crush is governed by $\sigma$ and the rate at which the material reaches full compaction is controlled by $n$. Other functional forms for $\lambda(p)$ may, however, be appropriate for other applications.

The $\mathrm{p}-\lambda$ compaction model requires the user to supply two parameters, the compaction strength $\sigma$ and the compaction hardening $n$. These parameters are needed for the 
$\mathrm{p}-\lambda$ crush model to describe the extent of compaction represented by Eq. (2) for a system of components within a mixture. The EBSC model was developed and intended to describe a mixture based solely on a small number of readily available component properties. Therefore, the $\sigma$ and $n$ parameters need to be derived from component level properties. An effective stress approach based on the original ideas of Terzaghi [4] offers a method for defining values of $\sigma$ and $n$ for a specific mixture.

\section{Determining Compaction Strength Parameters}

Some guidelines for defining the compaction parameters $\sigma$ and $n$ that make up the $\mathrm{p}-\lambda$ compaction model from component properties are beginning to emerge. For example, the compaction strength $\sigma$ is expected to scale with a measure of the inherent component strengths and the range of the compaction hardening $n$ appears from experiment to be bounded by the level of the mixture distension.

The method of determining the $\mathrm{p}-\lambda$ compaction strength parameter is still under development and is currently based on the principles originating from the effective stress theory of Terzaghi [4] and Skempton [5]. The effective stress concept of Terzaghi quoted that; "All the measurable effects of a change in stress, such as compression, distortion, and a change in shearing resistance, are exclusively due to changes in effective stress." The concept has been very successful in describing the observed settlement and the compaction behavior of saturated granular soils. The expression for effective stress given by Terzaghi is,

$\sigma_{i j}^{e}=\sigma_{i j}-p \delta_{i j}$

where $\sigma_{i j}$, is the total stress, $p$, is the pore pressure, and $\delta_{i j}$ is the Kroenecker's delta. Terzaghi established this relation through experimental observation. Skempton [5] expanded the experimental work of Terzaghi in an attempt to use material compressibility to better describe the state of effective stress. Skempton's effective stress relation is,

$$
\sigma_{i j}^{e}=\sigma_{i j}-p\left(1-\frac{K_{s k}}{K_{g}}\right) \delta_{i j},
$$

where $K_{s k}$ and $K_{g}$ are the bulk moduli of the skeleton and the entrained grains of the mixture respectively. Skempton uses a modified form of the Terzaghi effective stress by applying a compliance factor to the pressure as seen in Eq. (4). Skempton's concept of using a compliance factor provides the motivation for determining the mixture compaction strength for application of the $\mathrm{p}-\lambda$ model.

The EBSC model assumes that the compaction parameter $\lambda$ depends on, at least to first order, volumetric stress $p$, component strengths $\tau_{i}$, component bulk moduli
$K_{i}$, and component volume fractions $\eta_{i}$. In this analysis a two parameter Weibull function of form,

$$
\lambda\left(p, \tau_{i}, K_{i}, \eta_{i}\right) \approx 1-e^{-(p / \sigma)^{n}},
$$

where $\sigma=f\left(\tau_{i}, K_{i}, \eta_{i}\right)$ and $n=f\left(\tau_{i}, K_{i}, \eta_{i}\right)$, attempts to relate the level of compaction $\lambda$ and it's independent variables through two Weibull parameters ( $\sigma$ - compaction strength) and ( $n$ - compaction hardening).

A two-state compaction transition theory is used to describe the compaction of a porous mixture by relating the bulk moduli and the inherent component strengths. This idea is schematically illustrated in Fig. 4. Consider a multicomponent system where each component has inherent material strength $\tau_{i}$, ambient bulk moduli $K_{i}$, and volume fraction $\eta_{i}$. Using a rule of mixtures, the iso-strain $K_{e}$ and iso-pressure $K_{p}$ zero pressure bulk moduli are,

$K_{e}=\sum_{i} \eta_{i} K_{i}$

and

$$
K_{p}=\left(\sum_{i} \frac{\eta_{i}}{K_{i}}\right)^{-1} .
$$

Also, using a deviator strength model described by Johnson [6], the intrinsic bulk material strength $\tau^{*}$ of the mixture is approximated by,

$$
\tau^{*}=\sum_{i} \tau_{i} \eta_{i}
$$

The iso-strain and iso-pressure moduli then can be arranged to compute a factor ( $\kappa$ ) similar to Skempton's compliance factor, namely,

$\kappa=1-\frac{K_{p}}{K_{e}}$.

With the factor $\kappa$ the $\mathrm{p}-\lambda$ compaction parameter is related to intrinsic material strength through,

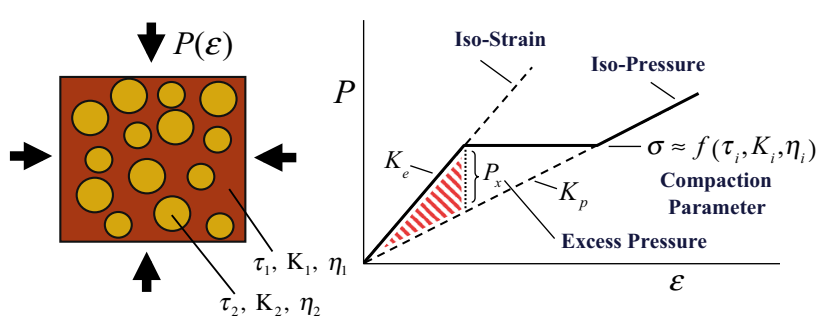

Fig. 4 A simplified two component system showing that the excess pressure above the equilibrium pressure (iso-pressure) $P_{x}$ contributes to the bulk compaction strength of the mixture. This pressure corresponds to the effective pressure of the mixture 
$\sigma=\frac{\tau^{*}}{\kappa}$

Secondly, the compaction-hardening factor $n$ is assumed to be a function of an extended definition of the mixture distension. This effective distension measure is calculated from the volume averaged and mass averaged densities of the mixture. The volume averaged and mass averaged densities of the mixture are,

$$
\begin{gathered}
\rho_{o o}=\sum_{i} \eta_{i} \rho_{i}^{o}, \\
\rho_{o}=\sum_{i} \mu_{i} \rho_{i}^{o} .
\end{gathered}
$$

The component mass fractions $\mu_{i}$ can be computed from the component volume fractions $\eta_{i}$ by the following ratio,

$$
\mu_{i}=\frac{\eta_{i} \rho_{i}^{o}}{\rho_{o o}} .
$$

The effective distension of the mixture is computed from the ratio of the mass averaged density to that of the volume averaged density,

$$
\alpha=\frac{\rho_{o}}{\rho_{o o}} .
$$

Note that this effective distension parameter reduces to the more familiar expression when a mixture of a solid and void is considered. A reasonable measure of the compaction hardening parameter $n$ has been observed from extensive compaction data to be approximated by,

$n=\frac{1}{\alpha}$.

\section{High-Pressure EOS (Isobaric Approach)}

The goal in this section is to develop an equation of state model that can predict the dynamic response of a heterogeneous mixture into a high pressure state. The Mie-Grïneisen EOS is commonly used for the high-pressure response of a fully compacted mixture. The Mie-Grüneisen EOS, however becomes awkward to use due to intense shock compression of highly distended materials. The pressure range of the model consists of the low-pressure mechanical crush of the mixture up to the extreme thermal states associated with shock compression of distended mixtures. This section offers an alternative description of the hydrodynamic iso-pressure boundary for the existing $\mathrm{p}-\lambda$ compaction model [1] described in Sect. 2 .

Extreme material heating due to intense shock compression and the associated volume expansion can lead to a backward leaning and multivalued Hugoniot response. This behavior is illustrated in Fig. 5. Recognizing the

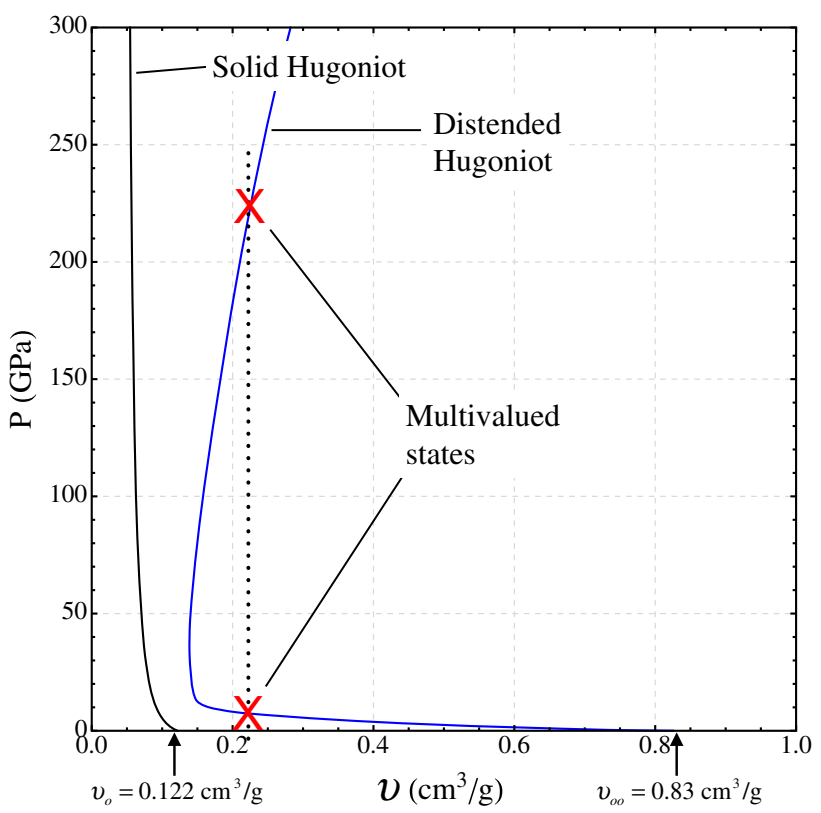

Fig. 5 Multivalued Hugoniot response of a distended mixture. This plot is a calculated Hugoniot for $\mathrm{Ta}_{2} \mathrm{O}_{5}$ based on experimental data with properties, $\rho_{o}=8.2 \mathrm{~g} / \mathrm{cm}^{3}, v_{o}=0.122 \mathrm{~cm}^{3} / \mathrm{g}$ and $\rho_{o o}=1.2 \mathrm{~g} / \mathrm{cm}^{3}$, $v_{o o}=0.83 \mathrm{~cm}^{3} / \mathrm{g}$

phenomenological nature of the model, application is intended strictly for interpolation within the range of available experimental data. The model is benchmarked to available data and intended for interpolation within the range of the data. The range of experimental data span thermodynamic states where thermal and lattice energies are comparable. The model approximates thermal states within a span of material behavior where even detailed physics-based theories are challenged.

The derivation of the thermodynamic EOS component of the EBSC model parallels that of the Mie-Grüneisen EOS, where the principal (or "cold") thermodynamic relation,

$\Gamma(v)=\left.v \frac{\partial p}{\partial E}\right|_{v}$,

is assumed to be a function of the specific volume only. Rather, start with the relation,

$\xi(p)=\left.p \frac{\partial v}{\partial H}\right|_{p}$,

where $\xi$ is the RW parameter (after Rice and Walsh [3]) and is assumed to depend only on the pressure. The RW parameter is a dimensionless measure of the increase in volume when thermal energy is added to a body at constant pressure. This alternative EOS development introduced by Rice and Walsh [3] and later expounded by [7] is joined 
with a principal (cold) Hugoniot as the reference relation between specific volume and pressure. Equation (16) is integrated from a reference specific volume $v_{R}(p)$ and reference enthalpy $H_{R}(p)$ to yield a relation for the specific volume as a function of pressure and enthalpy,

$v(p, H)=v_{R}(p)+\frac{\xi(p)}{p}\left(H(p)-H_{R}(p)\right)$.

Equation (17) is analogous to the Mie-Grüneisen EOS obtained when Eq. (4) is integrated holding volume constant from a reference pressure and corresponding reference energy. The Rice-Walsh parameter $\xi$ and the Grüneisen $\Gamma$ are related through,

$\xi(p)=\frac{\Gamma(p) p}{K_{s}(p)}$,

where the Grüneisen gamma $\Gamma(p)$ and isentropic bulk modulus $K_{s}(p)$ are evaluated at pressure on the reference Hugoniot. Rice and Walsh [3] developed an equation of state for water based on an assumption that $\xi$ is only a function of pressure. The functional form for the Grüneisen gamma in the present EOS development is based on equation of state work of Altschuler [8]. Namely, the ambient Grüneisen gammas $\gamma^{o}$ of the mixture components are known from their ambient thermodynamic properties, resulting in an ambient mixture Grüneisen gamma $\Gamma_{o}$ determined by the assumed additive mixture theory relation,

$\Gamma_{o}=\frac{1}{\rho_{o o}} \sum_{i} \eta_{i} \rho_{i}^{o} \gamma_{i}^{o}$,

while at very high quantum mechanical pressures the bulk mixture Grüneisen gamma approaches a sufficiently accurate theoretical minimum [9] of $\Gamma_{\infty}=0.5$. This minimum is based on a statistical Thomas-Fermi model, which approximates the distribution of electrons in the atoms of a material. The $\eta_{i}, \rho_{i}^{o}$, and $\gamma_{i}^{o}$ in Eq. (19) represent the $i$ th component volume fraction, ambient density, and ambient component Grüneisen gamma respectively.

A physically reasonable approximation of intermediate states for the Grüneisen gamma based on the work of Altschuler [8] is provided by the pressure-dependent function,

$$
\Gamma(p)=\Gamma_{o} e^{-\left(p / K_{o}\right)}+\Gamma_{\infty}\left(1-e^{-\left(p / K_{o}\right)}\right),
$$

where the pressure $p$ is normalized by the initial bulk modulus of the mixture. Figure 6 shows the pressure-dependent gamma for a porous mixture of powdered $\mathrm{Ta}_{2} \mathrm{O}_{5}$ (tantalum pentoxide) and air over a pressure range from 0 to $500 \mathrm{GPa}$. As pressure increases the Grüneisen gamma function approaches the value set for $\Gamma_{\infty}$. Using the work of Altschuler [8] as a guide, we set $\Gamma_{\infty}=0.8$, which works well over this pressure range for $\mathrm{Ta}_{2} \mathrm{O}_{5}$. Figure 6 also shows Eq. (20) plotted with the constant gamma $\left(\Gamma(p)=\Gamma_{o}\right)$ and constant

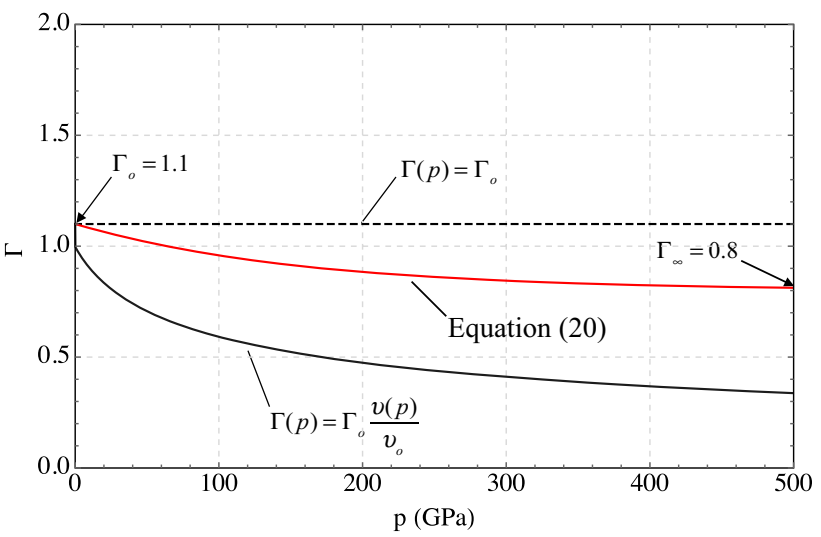

Fig. 6 Grüneisen gamma over a large pressure range for the $\mathrm{Ta}_{2} \mathrm{O}_{5}$ mixture, where the parameters in Eq. (18) are $K_{o}=86.8 \mathrm{GPa}$, $\Gamma_{o}=1.1$, and $\Gamma_{\infty}=0.8$

rho-gamma $\left(\rho(p) \Gamma(p)=\rho_{o} \Gamma_{o}\right)$ approximations to illustrate the bounds of Eq. (20) for the $\mathrm{Ta}_{2} \mathrm{O}_{5}$ material.

The isentropic bulk modulus $K_{s}(p)$ in Eq. (18) is sensibly estimated from the slope of the Hugoniot at a pressure state through appropriate thermodynamic relations [10]. Assuming a linear shock velocity versus particle velocity equation of state,

$p=\frac{\rho_{o} C_{o}^{2} \epsilon(p)}{(1-S \epsilon(p))^{2}}, \quad$ where $\epsilon(p)=1-v(p) / v_{o o}$,

and applying the Hugoniot derivative $K_{h} \equiv-v d p / d v=$ $(1-\epsilon) d p / d \epsilon$ to Eq. (21) yields,

$K_{h}(p)=\rho_{o} C_{o}^{2} \frac{(1-\epsilon(p))(1+S \epsilon(p))}{(1-S \epsilon(p))^{3}}$.

The isentropic bulk modulus as a function of pressure is,

$K_{s}(p)=K_{h}(p)\left[1-\frac{\Gamma(p) S \epsilon(p)^{2}}{(1-\epsilon(p))(1+S \epsilon(p))}\right]$,

where the dependence on pressure is shown to emphasize the bulk modulus versus pressure relation requisite to the Rice-Walsh form of the equation of state.

To calculate the Hugoniot states in distended materials using the Rice and Walsh EOS it is convenient to reference from the principal Hugoniot of the solid material. Therefore, the respective specific enthalpies shown in Eq. (17) are,

$$
\begin{aligned}
H_{R}(p) & =\frac{1}{2} p\left(v_{o}+v_{R}(p)\right) \\
H(p) & =\frac{1}{2} p\left(v_{o o}+v(p)\right) .
\end{aligned}
$$

Substituting Eqs. (18) and (24) into Eq. (17) yields the Hugoniot specific volume as a function of pressure. 
This expression is the Rice and Walsh EOS for the initially distended material,

$v_{h}(p)=v_{R}(p)+\frac{v_{o o}-v_{o}}{\frac{2 K_{s}(p)}{\Gamma(p) p}-1}$,

where $v_{R}(p)$ is the reference Hugoniot. This analysis uses a principal Hugoniot as a reference, but an isotherm or an isentrope could equally serve as a valid reference. The principal Hugoniot reference condition is preferred in this analysis because a linear shock-velocity versus particlevelocity EOS is commonly used and provides easy access to EOS parameters for the individual components of a porous mixture, e.g., $C_{o}$ the bulk sound speed, $S$ the linear slope, and $\rho_{o}=v_{o}^{-1}$ the initial solid material density.

When combined with the Hugoniot conservation relations an analytic relation for the reference Hugoniot pressure as a function of specific volume is obtained,

$p\left(v_{R}\right)=\frac{\rho_{o} C_{o}^{2}\left(1-v_{R} / v_{o}\right)}{\left(1-S\left(1-v_{R} / v_{o}\right)\right)^{2}}$.

Equation (26) can be inverted to provide an expression for the reference principal Hugoniot specific volume as a function of pressure,

$v_{R}(p)=v_{o}-v_{o} \frac{\alpha(p)}{S}\left(1-\sqrt{1-\alpha(p)^{-2}}\right)$

where

$$
\alpha(p)=1+\frac{\rho_{o} C_{o}^{2}}{2 S p} .
$$

The second term on the right in Eq. (25) represents the thermal specific volume change from the reference specific volume for a given initial bulk specific volume $v_{o o}$ and initial solid specific volume $v_{o}$. Equation (25) is the form derived from the Rice and Walsh EOS approach used in this analysis. Substituting the expressions given by Eqs. (20), (22), and (27) into Eq. (25) yields a Hugoniot relation for shock compression of initially distended materials. The fully compacted mixture material is considered to be in pressure and temperature equilibrium.

This equation of state offers a reasonable representation of high thermal expansion response that is present in many distended materials when subjected to intense shock states. This capability will be presented with some detail in Sect. (6) of this article where specific materials are modeled.

\section{Temperature of Shock Compression}

Shock induced heating and the accompanying temperature rise in the shock wave is a complex non-equilibrium event. On the continuum scale, temperature rise in a structured shock wave is near adiabatic with thermal conduction occurring on time scales far larger than that of wave propagation. The local scale shock heating, however, is far from adiabatic. Heterogeneous dissipation and thermal conduction are competing effects on the micro-scale that are often first-order influences on physical, chemical, and metallurgical processes occurring in the shock. Despite such complexities, sensible calculations of temperature based on continuum thermodynamic principles are tractable and provide useful bounds on the shock-heating event. Temperature states achieved in shock loading can be assessed through application of continuum thermodynamic principles. Shock temperature relations are developed with emphasis on distended granular solids.

The rise in the continuum temperature of a solid subjected to shock compression is calculated through fundamental thermodynamic relations. Temperature increase on the Hugoniot is determined through manipulation of the differential form of the specific enthalpy,

$d H=T d S+v d p$.

Symbols are respectively the system enthalpy, temperature, entropy, volume, and pressure. The temperature-pressure differential of the entropy is,

$d S=\left.\frac{\partial S}{\partial T}\right|_{p} d T+\left.\frac{\partial S}{\partial p}\right|_{T} d p$.

Extensive parameters $(H, S, v)$ are here considered specific values (per unit mass) although this is not required. Through the definition of the specific heat and the appropriate Maxwell relations,

$$
\begin{aligned}
& \left.\frac{\partial S}{\partial T}\right|_{p}=\frac{c_{p}}{T}, \\
& \left.\frac{\partial S}{\partial p}\right|_{T}=-\left.\frac{\partial v}{\partial T}\right|_{p}=-\left.c_{p} \frac{\partial v}{\partial H}\right|_{p}=-c_{p} \frac{\xi}{p} .
\end{aligned}
$$

Equation (30) reduces to,

$$
d S=\frac{c_{p}}{T} d T-\frac{c_{p} \xi}{p} d p
$$

The parameter $\xi$ is the material thermodynamic RW parameter (see Eq. 18).

The following formalism can be used to calculate the Hugoniot temperature of an initially distended or porous material. Assuming complete compaction within the shock wave, and that shock dissipation is fully converted to thermal energy uniformly distributed over the lattice, the specific enthalpy can be differentiated on the porous Hugoniot resulting in, 
$d H=\frac{1}{2}\left(v_{o o}+v(p)\right) d p+\frac{1}{2} p d v$.

Enthalpy and entropy are eliminated by substituting Eqs. (32) and (33) into Eq. (29), which results in a temperature differential on the Hugoniot,

$\frac{d T}{d p}-\frac{\xi(p)}{p} T=\frac{1}{2 c_{p}}\left[p \frac{d v}{d p}+\left(v_{o o}-v(p)\right)\right]$.

Equation (34) is the Hugoniot temperature differential with respect to pressure for a distended material. The expression for $\xi(p)$ is given by Eq. (18), the specific volume $v(p)$ and its derivative $d v / d p$ are determined from Eq. (25), and $v_{o o}$ is the initial specific volume of the bulk material. The variability of temperature rise due to a materials initial bulk density is illustrated in Fig. 7.

To compute the various Hugoniot curves an initial temperature $T_{0}$ at $P=0 \mathrm{GPa}$ is taken as $T_{0}=298 \mathrm{~K}$. Although certainly insufficient, a constant specific heat for $\mathrm{Ta}_{2} \mathrm{O}_{5}$ of $c_{p}=306.06 \mathrm{~J} /(\mathrm{kg} \mathrm{K})$ as reported by Samsonov [11] is used. This specific heat is within the Dulong-Petit limit of $395.13 \mathrm{~J} /$ $(\mathrm{kg} \mathrm{K})$. The nearly flat curve is the melt line of solid $\mathrm{Ta}_{2} \mathrm{O}_{5}$ assuming a Lindemann melt law [12] with an upper-bound initial melt temperature of $2088 \mathrm{~K}$ as reported by Samsonov [11]. The melt curve is calculated in order to provide a reference for the range of shock temperatures generated. The temperatures and pressures of the lower initial density materials, (e.g., $\rho_{o o}=1.2 \mathrm{~g} / \mathrm{cc}$ and $\rho_{o o}=3.0 \mathrm{~g} / \mathrm{cc}$ ), at computed Hugoniot densities reveal that the material states are entering a region identified as that of warm dense matter (WDM) [e.g., Drake [13]].

\section{Isentropic Decompression}

The common shock-wave event usually consists of a strong but brief pressure loading followed by a more gradual release of pressure on decompression from the

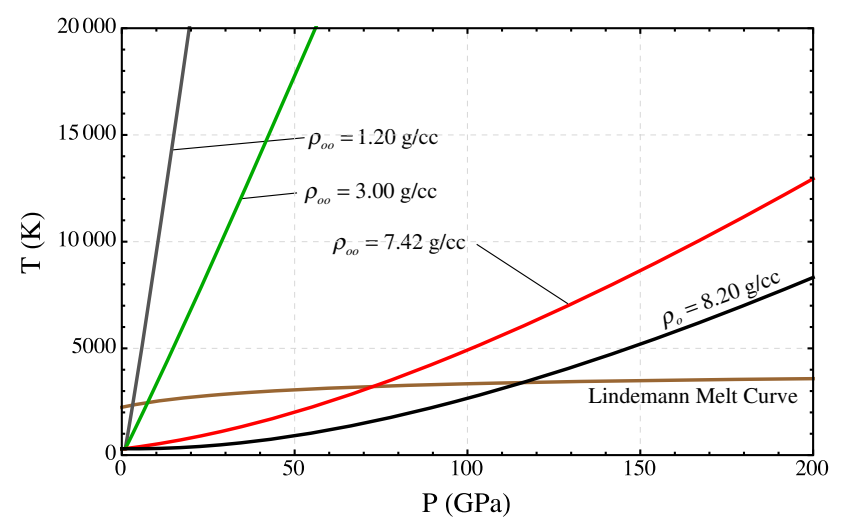

Fig. 7 Hugoniot temperature calculations using Eq. (34) for various initial bulk densities of $\mathrm{Ta}_{2} \mathrm{O}_{5}$ powder. The solid $\mathrm{Ta}_{2} \mathrm{O}_{5}$ Hugoniot $\left(\rho_{o}=8.20 \mathrm{~g} / \mathrm{cc}\right)$ and the Lindemann melt curve are also plotted shock state. This process in a material is usually modeled as shock compression along a Rayleigh line to a Hugoniot state followed by isentropic decompression. This concept of the shock event is illustrated in Fig. 8.

Analysis of the isentropic release response of a material from a Hugoniot state can be accomplished through application of principles from shock-wave equation-of-state thermodynamics. The equation of state proposed by Rice and Walsh [3] starts with the thermodynamic differential given by Eq. (16) and is assumed to be only pressure dependent. Integrating and holding pressure constant from some reference specific volume and reference enthalpy, results in the Rice-Walsh form of the equation of state as given by Eq. (17). In this instance, $\frac{\xi(p)}{p}=\eta(p)$ is the pressure dependent property,

$\eta(p)=\frac{\xi(p)}{p}=\left.\frac{\partial v}{\partial H}\right|_{p}=\frac{\Gamma(p)}{K_{s}(p)}$.

This relation is named the Rice-Walsh factor and has units of inverse pressure. The Rice-Walsh factor is the ratio of the materials Grüneisen gamma and the isentropic bulk modulus known from the reference equation of state.

Solution of the Rice-Walsh equation of state leads to the following expression for the isentropic decompression relation centered at any shock Hugoniot state. The specific volume at any pressure is composed of the cold compression and the thermal expansion. The Hugoniot enthalpy for the distend material,

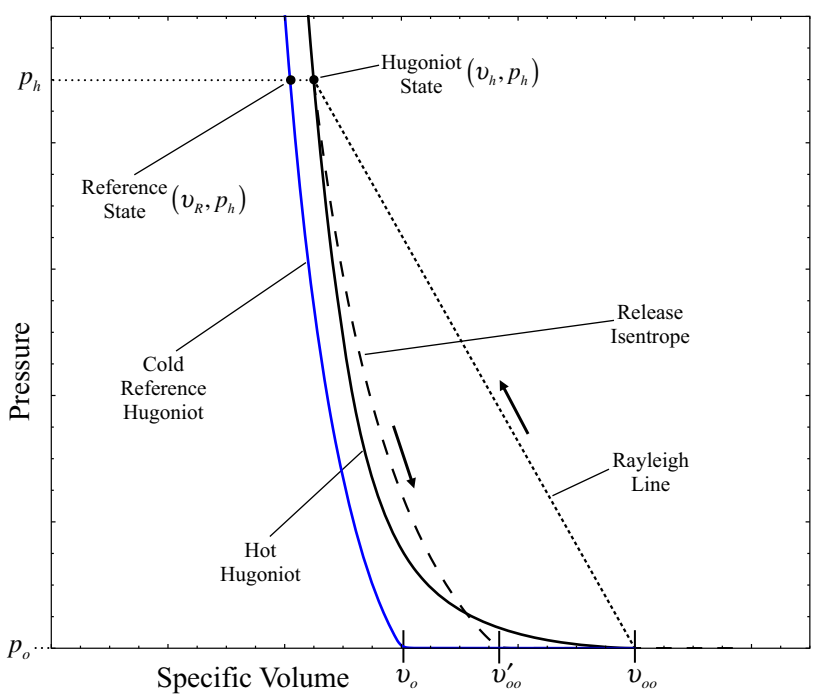

Fig. 8 Rayleigh-line shock loading from $\left(v_{o o}, p_{o}\right)$ to a Hugoniot state $\left(v_{h}, p_{h}\right)$ followed by pressure release along an isentropic path back down to $\left(v_{o o}^{\prime}, p_{o}\right)$ 
$H(p)=\frac{1}{2} p\left(v_{o o}+v(p)\right)$,

and the Rice-Walsh EOS in Eq. (17) provide a relation for the Hugoniot centered at the distended specific volume $v_{o o}$,

$$
\begin{aligned}
v_{h}(p)= & v_{R}(p) \\
& +\frac{\eta(p)}{1-\frac{1}{2} p \eta(p)}\left(\frac{1}{2} p\left(v_{o o}+v_{R}(p)\right)-H_{R}(p)\right) \\
= & v_{R}(p)+v_{t h}(p) .
\end{aligned}
$$

Full compaction to the EOS surface is assumed. The second term $v_{t h}(p)$ in Eq. (37) is the thermal specific volume at the Hugoniot state.

To obtain the decompression isentrope the principal Hugoniot is used as defined by Eq. (27). The principal Hugoniot is identified as that of a porous material in the limit of zero porosity; namely the ambient solid Hugoniot. The reference equation of state $v_{R}(p)$ is the cold volume accounting for the lattice compression at absolute zero temperature. Data for the ambient solid Hugoniot of many materials [14] is usually easy to obtain.

Starting with Eq. (17), rearranging the expression, and defining the differences to represent the thermal specific volume and thermal specific enthalpy, respectively,

$$
\begin{aligned}
v_{h}(p)-v_{R}(p) & =\eta(p)\left(H-H_{R}(p)\right) \\
v_{t h}(p) & =\eta(p) H_{t h}(p) .
\end{aligned}
$$

Differentiate Eq. (38) with respect to pressure holding entropy constant,

$$
\left.\frac{\partial v_{t h}(p)}{\partial p}\right|_{S}=\frac{d \eta(p)}{d p} H_{t h}(p)+\left.\eta(p) \frac{\partial H_{t h}(p)}{\partial p}\right|_{S},
$$

the following first order homogeneous differential equation is obtained,

$\frac{d v_{t h}(p)}{d p}-\left(\frac{\eta^{\prime}(p)}{\eta(p)}+\eta(p)\right) v_{t h}(p)=0$.

Solving Eq. (40) provides the isentrope centered at the Hugoniot state $\left(p_{h}, v_{h}\left(p_{h}\right)\right)$,

$v_{S}(p)=v_{R}(p)+v_{t h}\left(p_{h}\right) \frac{\eta(p)}{\eta\left(p_{h}\right)} e^{\int_{p_{h}}^{p} \eta\left(p^{*}\right) d p^{*}}$.

The specific volume at any pressure on the decompression isentrope is decomposed into the cold compression and the thermal expansion. The first term in Eq. (41) is the cold compression Hugoniot. The second term accounts for the thermal expansion due to shock heating up to the Hugoniot state $v_{h}\left(p_{h}\right)$. The Rice-Walsh factor $\eta(p)$ is given by Eq. (35). This analysis uses Eq. (20) to represent the
Grüneisen gamma function $\Gamma(p)$ and Eq. (23) for the isentropic bulk modulus.

\section{Applying the Model}

Tungsten carbide (WC), tantalum pentoxide $\left(\mathrm{Ta}_{2} \mathrm{O}_{5}\right)$, and water-saturated calcite $\left(\mathrm{CaCO}_{3}\right)$ were selected as test materials to demonstrate the abilities of the EBSC EOS model. These materials offer importance to industry and the shock-physics research community. Even though these materials have broad applications, the extreme high-pressure EOS at various distensions has not been widely studied and reported. However, recent experimental work conducted by Miller et al. [15] and Vogler et al. [16] on $\mathrm{Ta}_{2} \mathrm{O}_{5}$ and $\mathrm{WC}$ is providing needed data for this EOS development effort. Lastly, an extensive experimental investigation of shock compression on carbonate materials performed by Kalashnikov et al. [17] has helped to show the applicability of the EBSC model in describing the response of a mixture (water-saturated calcium carbonate).

\section{Shock Loading of Porous WC}

Tungsten carbide (WC) is representative of a class of materials that is high in bulk modulus and surface hardness. Through the application of the EBSC EOS utilizing the p- $\lambda$ material crush framework and the RW high-pressure EOS, along with support from collected experimental data, a reliable EOS prediction over a large pressure range can be obtained and verified. We have compared results from analytical calculations, and experimental data based on distended WC $[16,18]$.

The EBSC EOS model applied to a porous WC powder through Eq. (25) is compared with experimental data in Fig. 9. The model is compared with data of Vogler et al. [16] at an initial distension of $\rho_{o} / \rho_{o o}=1.82$. Experimental data from Trunin et al. [18] is used to generate the reference Hugoniot. The log-linear plot of Fig. 9b illustrates the EBSC model's ability to represent the low-pressure (0-5 GPa) crush and subsequent high-pressure (50$500 \mathrm{GPa}$ ) end-states of the porous WC. The EBSC model uses the concepts described in Sect. 2 to represent the material crush and subsequent transition into the highpressure equilibrium response (Sect. 3) of the porous WC material.

\section{Shock Loading of Porous $\mathrm{Ta}_{2} \mathrm{O}_{5}$}

Tantalum pentoxide $\left(\mathrm{Ta}_{2} \mathrm{O}_{5}\right)$ was also selected to test the EBSC EOS model. Using the Rice-Walsh EOS as the highpressure equilibrium EOS and the $\mathrm{p}-\lambda$ framework to address the low-pressure crush, a reasonable EOS prediction 


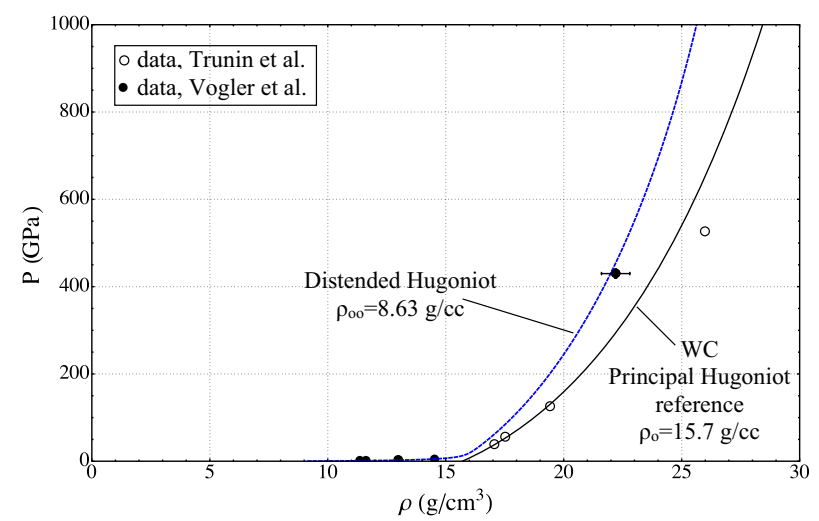

(a) Linear plot highlights model agreement with single high-pressure data point with error bars $\left( \pm 0.6 \mathrm{~g} / \mathrm{cm}^{3}\right.$ and $\pm 10.0 \mathrm{GPa})$.

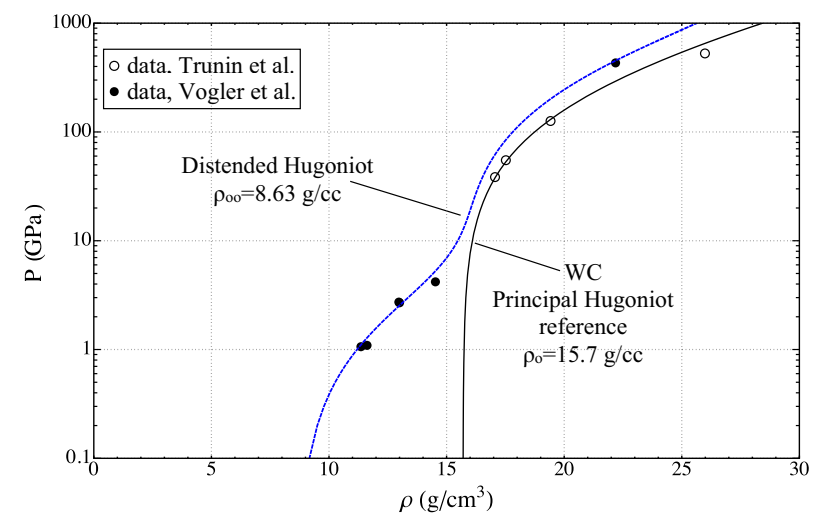

(b) Log-Linear plot displays low-pressure data and model agreement.

Fig. 9 Distended WC powder comparison of calculated model to experimental data

over a large pressure range is obtained. Figure 10 illustrates the EBSC EOS model description of the response at several initial distensions.

Hugoniot curves for six different initial distensions of $\mathrm{Ta}_{2} \mathrm{O}_{5}$ are shown in a log-linear plot. Both low-pressure crush and extreme pressure Hugoniot data are highlighted. The experimental data are from Miller et al. [15] and Vogler et al. [16, 19]. The Hugoniot reference curve for $\mathrm{Ta}_{2} \mathrm{O}_{5}$ is estimated from the $90 \%$ density Hugoniot data. This reference relation is described by an initial bulk modulus of $K_{o}=86.8 \mathrm{GPa}$ and a $K_{o}^{\prime}=3.0$ for solid $\mathrm{Ta}_{2} \mathrm{O}_{5}$. The initial bulk modulus $\left(K_{o}\right)$ was determined for $\mathrm{Ta}_{2} \mathrm{O}_{5}$ using micro-indentation techniques [20], since no high-pressure data on fully dense $\mathrm{Ta}_{2} \mathrm{O}_{5}$ was available. The log-linear plot ranges over 0.1-300 GPa in excess of three decades of pressure. This range of pressure was sufficient to demonstrate the low-pressure material crush and the equilibrium high-pressure response of the model.

The EBSC model inputs for all material results shown in Fig.10 are not quite universal in the use of the $\Gamma(p)$,

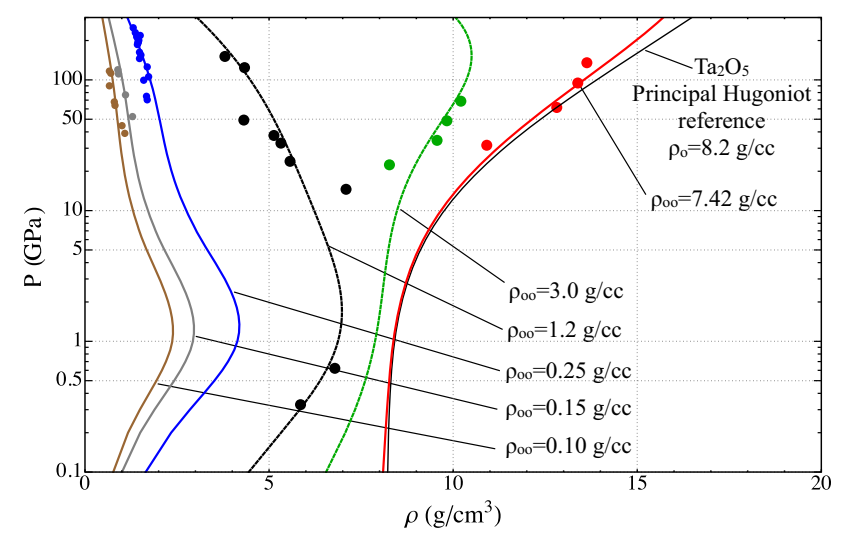

Fig. 10 Comparison of EBSC model to experimental data for $\mathrm{Ta}_{2} \mathrm{O}_{5}$. The reference curve for $\mathrm{Ta}_{2} \mathrm{O}_{5}$ is based on the bulk modulus $\left(K_{o}=86.8 \mathrm{GPa}\right)$ and an assumed value for the pressure derivative of the bulk modulus $\left(K_{o}^{\prime}=3.0\right)$

Eq. (20). For example, a constant $\Gamma(p)$, (i.e., $\Gamma_{o}=$ $\left.\Gamma_{\infty}=1.1\right)$ was used for the $\rho_{o o}=1.2 \mathrm{~g} / \mathrm{cc}$ material. This choice of $\Gamma(p)$ allowed a better fit of the model to the experimental data. Otherwise, for the $\rho_{o o}=1.2 \mathrm{~g} / \mathrm{cc}$ material, the model did not account for enough material expansion at higher pressures to fit the data. All other material dissensions used a smoothly varying $\Gamma(p)$, with a $\Gamma_{o}=1.1$ and a $\Gamma_{\infty}=0.8$.

\section{Shock Loading of Calcium Carbonate and Water}

In 1973, extensive experimental investigation of shock compression of carbonate materials was performed by Kalashnikov et al. [17]. This investigation focused on Hugoniot measurements on carbonate mixtures in initial dry-porous and water-saturated states. Calcite $\left(\mathrm{CaCO}_{3}\right)$ in the solid state (limestone with initial density of $2.665 \mathrm{~g} / \mathrm{cm}^{3}$, slightly less than the crystal density of $2.71 \mathrm{~g} / \mathrm{cm}^{3}$ ) and calcite-water mixture at $13.6 \%$ by weight was chosen to compare with the EBSC model.

This comparison demonstrates the models ability to predict the shock response of heterogeneous component mixtures. Figure 11 shows a comparison of the EBSC EOS model with the Kalashnikov et al. [17] Hugoniot data and water Hugoniot data from Marsh [14]. Both the pressuredensity and shock-velocity-particle-velocity plots are presented.

\section{Summary}

The EBSC equation of state model is useful for demonstrating porous mixture behavior over a range from mechanical compaction to extreme pressures. It takes advantage of the volume superposition of two reasonably 
Fig. 11 Shock Hugoniot data for water $\left(\rho_{o}=0.998 \mathrm{~g} / \mathrm{cm}^{3}\right)$, water-saturated calcite

$\left(\rho_{o o}=2.20 \mathrm{~g} / \mathrm{cm}^{3}\right)$, and solid calcite (limestone

$\left.\rho_{o}=2.665 \mathrm{~g} / \mathrm{cm}^{3}\right)$. The dashed line in both plots is the model representation for the calcitewater mixture

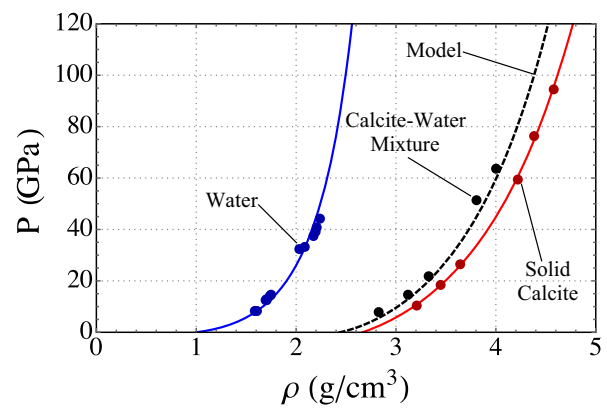

(a) Pressure vs. density plot.

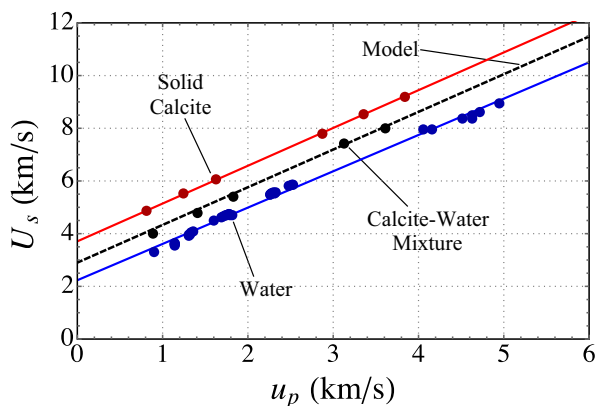

(b) Linear shock-velocity vs. particle-velocity plot. well-characterized compression regimes, namely the isostrain and iso-pressure regimes. These regimes are elastic compression relations for and the initially distended media (iso-strain) and the fully compacted solid media (isopressure). A pressure dependent function ranging from 0 for the initial uncompacted media to 1 for the fully compacted material is used to model the compaction of the material. The guidelines for determining the EBSC models compaction parameters, which define the EBSC crush response was described. The details on the guidelines are based on the effective stress theories of Terzaghi [4], Skempton [5], and a deviator strength model described by Johnson [6].

Once full compaction is achieved, an isobaric enthalpybased equation of state represents the iso-pressure condition of the mixture and subsequent isentropic release from the Hugoniot state. The EBSC model uses an enthalpybased equation of state to avoid awkward multivalued Hugoniot states due to intense shock compression of highly distended materials and extreme material heating and associated volume expansion. Rice and Walsh [3] initially introduced this seminal thermodynamic approach for the purpose of a complete thermodynamic description of water at high-pressure. A thermodynamic parameter emerges and is called the Rice-Walsh parameter. This parameter is described and related to the Grüneisen gamma and the isentropic bulk modulus. Both the Grüneisen gamma and the isentropic modulus are assumed to be singular in dependence to pressure, and therefore allowing the RiceWalsh parameter to be solely a function of pressure. The adopted functional dependence of the Grüneisen gamma is based on the equation of state work of Altschuler [8].

The shock-wave compression event usually consists of a strong but brief pressure loading followed by a more gradual release of pressure on decompression from the shock state. This shock process is typically acquired along a Rayleigh line to a Hugoniot state followed by isentropic decompression. However, this process is only approximate, but it turns out to be a very good approximation. The analysis of the isentropic release of a material from a
Hugoniot shock state are described and derived through the application of principles of shock-wave equation-of-state thermodynamics.

The EBSC model is compared against experimental Hugoniot data for porous tungsten carbide, porous tantalum pentoxide $\left(\mathrm{Ta}_{2} \mathrm{O}_{5}\right)$, and a mixture of calcite and water $\left(\mathrm{CaCO}_{3}\right)$. These materials are attractive materials for investigating shock equation-of-state features of distended mixture compounds because they represent mixture materials with disparate components and are materials with good Hugoniot data. Vogler et al. [16] have established extreme-pressure Hugoniot properties for several different initial powder configurations, as well as for a near-fulldensity WC (10\% porosity) sintered ceramic. The data for WC represents some of the highest pressure data reported in the literature for distended materials, while the data for $\mathrm{Ta}_{2} \mathrm{O}_{5}$ reaches high pressures for two initial densities and a nearly full-consolidated form of the material. The data on $\mathrm{Ta}_{2} \mathrm{O}_{5}$ is complemented by experiments from the literature [15] utilizing lasers that were performed on aerogels at three initial densities.

This enthalpy-based shock modeling approach appears to reasonably reflect the equation of state response of porous mixture materials at various initial distensions. This material model has received limited but successful application. The model is evolving and will continue to undergo change. The EBSC model is intended to be a robust computational model with relatively few parameters to describe the compression response of a mixture material.

Acknowledgments The authors gratefully acknowledge Sandia National Laboratories for the financial support Granted under Contract PO 861392 without which this effort would not have been possible.

\section{References}

1. Grady D, Winfree N, Kerley G, Wilson L, Kuhns L (2000) Computational modeling and wave propagation in media with inelastic deforming microstructure. In: Proceeding of the DYMAT 2000, 6th international conference on mechanical and 
physical behaviour of materials under dynamic loading, vol 10 . EDP Sciences, Les Ulis, pp 15-20

2. Grady D (2001) P-lambda model for material mixtures: application to compaction of porous zirconium dioxide. Technical report, Applied Research Associates

3. Rice MH, Walsh JM (1957) Equation of state of water to 250 kilobars. J Chem Phys 26(4):824

4. von Terzaghi K (1936) The shear resistance of saturated soils. In: Proceedings of the 1st international conference on soil mechanics and foundation engineering, vol. 1. Harvard University Press Cambridge, pp 54-56

5. Skempton AW (1960) Effective stress in soils, concrete and rocks. In pore pressure and suction in soils. Butterworth, Oxford, pp 4-16

6. Johnson JN (1979) Analytic failure surfaces for oil shales of varying kerogen content. In: Timmerhaus K, Barber M (eds) High pressure science and technology, vol 2. Springer, New York, pp 1476-1486

7. Wu Q, Jing F (1996) Thermodynamic equation of state and application to hugoniot predictions for porous materials. J Appl Phys 80:4343

8. Altschuler L, Sharipdzhanov I (1971) Additive equation of state of silicates at high pressure. Phys Solid Earth 3:167

9. Zel'dovich YB, Raizer YP (2002) Physics of shock waves and high-temperature hydrodynamic phenomena. Dover Publications Inc., New York, pp 703-704

10. Duvall GE, Fowles GR (1963) High pressure physics and chemistry, vol. 2, Chap. 9. Academic Press, Waltham, pp 215-219
11. Samsonov GV (1982) The oxide handbook, 2nd edn. IFI/Plenum, Kiev

12. Lindemann FA (1910) The calculation of molecular vibration frequencies. Phyzik Z 11:609

13. Drake RP (2010) High-energy-denisty physics. Phys Today 63(6):28

14. Marsh SP (ed) (1980) LASL shock hugoniot data. University of California Press, Oakland

15. Miller J, Boehly T, Meyerhofer D, Eggert J (2007) Equation-ofstate measurements in $\mathrm{Ta}_{2} \mathrm{O}_{5}$ aerogel. In: SCCM-2007: AIP Conference Proceedings. Waikoloa, Hawaii, pp 71-74

16. Vogler TJ, Root S, Knudson MD, Reinhart WD (2011) Highpressure shock behavior of $\mathrm{WC}$ and $\mathrm{Ta}_{2} \mathrm{O}_{5}$ powders. Technical Report SAND2011-6770. Sandia National Laboratories, Albuquerque, New Mexico 87185, Livermore, California 94550 (2011)

17. Kalashnikov N, Pavlovskiy M, Simakov G, Trunin R (1973) Dynamic compressibility of calcite group minerals. Phys Solid Earth 2:80

18. Trunin R, Gudarenko M, Zhernokletov, Simakov G (2001) Experimental data on shock compression and adiabatic expansion of condensed matter, vol 1, 1st edn. Russian Federal Nuclear Center-VNIIEF, Sarov

19. Vogler T (2010) Personal conversations

20. Buchheit TE, Vogler TJ (2010) Measurement of ceramic powders using instrumented indentation and correlation with their dynamic response. Mech Mater 42:599 